\title{
Rendering the Unfolded Cerebral Cortex
}

\author{
Junfeng Guo ${ }^{1}$, Alexandru Salomie ${ }^{2}$, Rudi Deklerck ${ }^{2}$, and Jan Cornelis ${ }^{2}$ \\ 1 Institute of IPPR \\ Shanghai Jiaotong University, 200030 Shanghai, China \\ 2 Dept. ETRO-IRIS \\ Vrije Universiteit Brussel, Pleinlaan 2, 1050 Brussels, Belgium
}

\begin{abstract}
Classical volume rendering is computed by casting a bundle of parallel rays from a flat viewing plane onto the volume data set, and produces as such a spatially limited view of the objects in the data set. The method described in this paper is able to generate an overall planar view of an object that is topologically compatible with the sphere, by firing rays from a nearby surrounding surface and by unfolding this surface in a $2 \mathrm{D}$ plane, without introducing major distortions. It has been devised to facilitate the interpretation of the cerebral cortex. An initial surface consisting of two hemi-ellipsoids, one to cover the top and another one to surround the bottom of the brain, is interactively defined and deformed via a deformable model approach towards a dilated version of the cortical surface of the brain. During deformation, the nodes on the surface are continuously redistributed, to maintain a near homothetic mapping with the plane. Once the surface has converged to the dilated brain surface, rays are casted from the nodes, according to the normal of the surface at the node. The shading result, computed at the intersection of the rays with the original brain surface, is mapped via the near homothetic mapping to the plane. With this approach sulci can be followed in their entirety, so that it is much easier to derive their spatial relationship and to recognize them.
\end{abstract}

\section{Introduction}

The idea of producing two-dimensional cortical maps dates from the seventies $[1,2]$. At that time, a purely manual approach was used to attempt preserving measures of length, angle or area. Later on, computerised methods have been designed: Dale and Sereno [3] apply an iterated smoothing of the cortical surface, that only preserves neighbourhood relationships on the cortex. The most powerful methods are designed to keep geometric distortions as small as possible. Optimally, one should try to construct an isometric mapping, between the cortical surface and the plane, but as known from differential geometry this mapping only exists between surfaces which have the same Gaussian curvature $K$. As the plane has a constant Gaussian curvature $K$ equal to zero and $K$ is quite variable for the cortical surface, it is only possible to make an approximate isometric mapping. 
Schwarz et al. $[4,5]$ start from a triangular mesh and try to optimally preserve the metric structure of this mesh, represented as a distance matrix with elements $(i, j)$ equal to the shortest distance along the edges of the mesh between the vertices $i$ and $j$. They use the Newton-Raphson method to maximise the goodness of fit between the distance matrix of the original surface and the distance matrix of the planar configuration. It is evident that this exhaustive calculation of all possible paths must lead to a good estimate of the global metrical characteristics of the surface, but this is achieved at the cost of an enormous computational load and combinatorial complexity. Therefore Schwartz et al. [4,5] divide the surface in overlapping patches of about 300 vertices.

Another solution to reduce the computational complexity, is to work only with first- or second-order nearest neighbour distances. However this approach is not always stable, as the normals of the patches may reverse in the planar configuration. In [6], this folding over of the facets in the plane is countered by introducing an additional error term in the residual energy functional. Since the distance matrix has now become a sparse matrix, a more efficient minimisation scheme based on the conjugate gradient approach can be devised.

Carman et al. [7] create two-dimensional maps of the cortex by modelling it as an elastic sheet whose internal forces act to preserve the topology and geometry of the sheet, while external forces act to unfold and flatten it. The unfolding force is designed to drive the overall surface into a planar configuration and to act against internal forces attempting to maintain intrinsic curvature or local folding. The internal forces are a longitudinal force and a torsional force which together model the elastic properties of the surface. The longitudinal force acts towards restoring the lengths between adjacent nodes of the triangular mesh to their reference values, while the torsional force attempts to preserve angles. All forces acting on a node $N$ are based on a geometric relationship with the immediate neighbours of the node $N$.

In all the previously mentioned approaches the initial mesh on the cortical surface is constructed without taking into account any relationship with the plane, where it will be flattened. The flattening technique used in this paper, which is based on the concept of homothetic mapping described in [8], directly starts from a plane and keeps track how the points in the plane are distributing themselves on the cortical surface after several steps of deformation. This approach has the additional advantage that a very uniform triangular mesh can be obtained after convergence. Furthermore, the flat map has a rectangular boundary, whereas the procedure followed by [9] generates flat maps with an arbitrary shape.

\section{Methods}

\subsection{Preprocessing, Segmentation}

A T1-weighted MR image set of the brain of sufficient resolution (e.g. 100 slices with a size of $256 \times 256)$ is segmented, i.e. all grey and white matter tissues 
are isolated via an approach based on the principles of seeded region growing, supervised clustering and morphological filtering[10]. The spinal cord is cut at the level of the bottom of the cerebellum. A dilated version of the binary volume is obtained by using an approximate spherical $7 \times 7$ mask.

\subsection{Definition of the Initial Surface}

Next, two hemi-ellipsoids are shaped interactively by adjusting their principle axes and common centre point, so that they fit as close as possible to the top and bottom parts of the brain. This interactive procedure is supported by a software, which provides three orthogonal views, arranged in a square four-window frame with the fourth window reserved for displaying the projection result. The axes of the ellipsoids are defined on the orthogonal sections of maximal brain extent.

Once the two hemi-ellipsoids are defined, the points $(u, v)$ of a rectangular regular grid, defined in a planar space (i.e. our plane of projection), are attributed a corresponding point $\mathbf{x}(u, v)$ on the ellipsoidal surface, in a way that the centre of the original rectangle is mapped to the top of the hemi-ellipsoid and the points lying on the perimeter of the grid are moved towards the perimeter at the bottom of the hemi-ellipsoid.

This transform wraps the planar sheet around the hemi-ellipsoid. Yet this wrapping causes the distances between the points on the surface to be no longer uniform. In order to obtain good projection results, the distances are homogenised for the first time by the iterative method, described further on in section 2.4. For the sake of the speed of convergence, we only map a coarse regular subsample of the points defined in the $(u, v)$ space to the hemi-ellipsoids. A fine-grain mapping is made, once the hemi-ellipsoid has deformed towards the dilated cortical surface. We find the hemi-ellipsoids leading to faster convergence and yielding better flattening results than the automatic approach based on hemispheres used in [8].

\subsection{Deformation Towards the Surface}

Once the points are homogenised, both the hemi-ellipsoidal surfaces are mapped towards the binary image of the brain (see Fig. 1) by means of a deformable surface model. The general formula of such a model is described in [11]:

$$
\begin{aligned}
& -\frac{\partial}{\partial u}\left(w_{10} \frac{\partial \mathbf{x}}{\partial u}\right)-\frac{\partial}{\partial v}\left(w_{01} \frac{\partial \mathbf{x}}{\partial v}\right)+2 \frac{\partial^{2}}{\partial u \partial v}\left(w_{11} \frac{\partial^{2} \mathbf{x}}{\partial u \partial v}\right) \\
& +\frac{\partial^{2}}{\partial^{2} u}\left(w_{20} \frac{\partial^{2} \mathbf{x}}{\partial^{2} u}\right)+\frac{\partial^{2}}{\partial^{2} v}\left(w_{02} \frac{\partial^{2} \mathbf{x}}{\partial^{2} v}\right)=\mathbf{F}(\mathbf{x})
\end{aligned}
$$

The coefficients $w_{i j}$ determine the mechanical properties of the surface. The elasticity (i.e. the stretching properties of the surface) is defined by $\left(w_{10}, w_{01}\right)$, while the rigidity (i.e. the effort needed to bend the surface) depends on $\left(w_{20}, w_{02}\right)$ and the resistance to twist is given by $w_{11}$. The term $\mathbf{F}(\mathbf{x})$ denotes the sum of external forces. When restricted to its first-order derivative terms, this model may be interpreted physically as a membrane, whereas the second order 
derivative terms model a thin plate. In order to simplify the computations, we prefer to omit the second order derivative terms and consider the surface acting as a membrane. In this way we obtain the following equilibrium equation, including the expanded external forces:

$$
\begin{aligned}
& \beta(u, v)\left[\mathbf{x}_{u u}(u, v)+\mathbf{x}_{v v}(u, v)\right] \\
& +[1-\gamma(u, v)] \cdot\left\{\mathbf{x}^{\prime}(u, v)-\mathbf{x}(u, v)\right\}+\gamma(u, v) N(u, v)=0
\end{aligned}
$$

where $\mathbf{x}^{\prime}$ is defined in Fig. 1 .

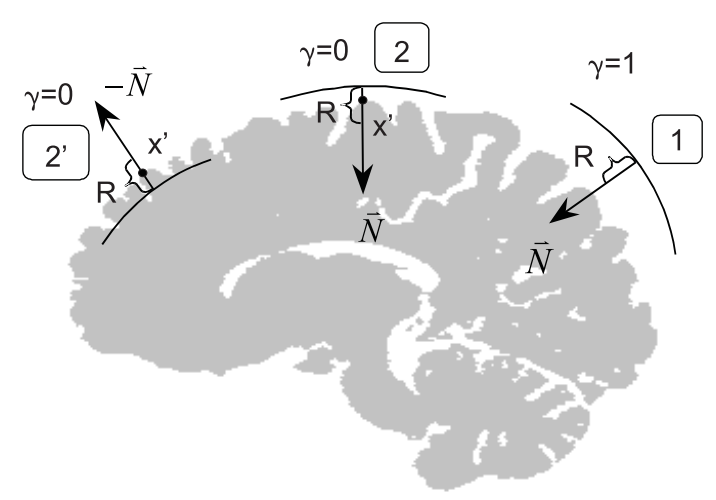

Fig. 1. External forces deforming the surface

In this model the elasticity is supposed to be isotropic and to be dependent on the position in the membrane: i.e. $w_{10}=w_{01}=\beta(u, v)$. A similar formula is used in [8], yet in their approach Davatzikos and Brian use a different restoring force - based on a centre of mass function - that makes the surface converge towards the centre of the cortical layer. In our approach, we are interested in retrieving the exterior surface of the cortex and therefore repulse the surface, once it enters the brain.

Instead of using a centre of mass function, that considers all the voxels within a certain spherical neighbourhood around a node $\mathbf{x}(u, v)$ and which makes only sense when there is a distinct grey-white matter border, we employ a modified and simpler computational method, by only scanning voxels along the normal direction within a certain distance $R$ from $\mathbf{x}(u, v)$. When the node $\mathbf{x}(u, v)$ is lying outside the brain and there is no intersection with the cortical surface found within the distance $R$ when scanning along the inner normal direction (case 1 in Fig. 1), an attractive force $\mathbf{F}_{\text {attr }}(\mathbf{x})$, acting along $\mathbf{N}(u, v)$ will be introduced: i.e. $\mathbf{F}_{a t t r}(\mathbf{x})=\gamma(u, v) \boldsymbol{N}(u, v)$ by setting $\gamma(u, v)=1$. As a consequence the restoring force $\mathbf{F}_{\text {rest }}(\mathbf{x})=(1-\gamma(u, v))\left(\mathbf{x}^{\prime}(u, v)-\mathbf{x}(u, v)\right)$ will be inactive in this situation. The force $\mathbf{F}_{\text {rest }}(\mathbf{x})$ can be either attractive or repulsive and will be made active by imposing $\gamma(u, v)=0$, when there is an intersection $\mathbf{x}^{\prime}(u, v)$. In case 2 , when the node $\mathbf{x}(u, v)$ is lying outside the brain, the restoring force will further attract 
the membrane towards the actual surface. In case $2^{\prime}$, the scanning occurs in the opposite direction and the derived restoring force will repel the node $\mathbf{x}(u, v)$. Similar to $\gamma(u, v), \beta(u, v)$ will take a different constant value when the node $\mathbf{x}(u, v)$ is close to the cortical surface (cases 2 and $2^{\prime}$ ) or far way (case 1 ), as explained in [8].

In order to solve equation 1 numerically, we can consider its evolution equation:

$$
\left\{\begin{array}{l}
\beta(u, v, t)\left[\mathbf{x}_{u u}(u, v, t)+\mathbf{x}_{v v}(u, v, t)\right]+[1-\gamma(u, v, t)] \cdot\left\{\mathbf{x}^{\prime}(u, v, t)-\mathbf{x}(u, v, t)\right\} \\
+\gamma(u, v, t) N(u, v, t)=\alpha(u, v, t) \mathbf{x}_{t}(u, v, t) \\
\mathbf{x}(u, v, 0)=\text { top or bottom hemi - ellipsoidal surface }
\end{array}\right.
$$

When the surface converges to its final position, $\alpha(u, v, t) \mathbf{x}_{t}(u, v, t)$ will tend towards zero, which also implies that the equilibrium equation 1 will be satisfied. By approximating the second order derivative with the finite difference formula $\mathbf{x}_{u u}^{t}(i, j)=\mathbf{x}_{i+1, j}^{t}-2 \mathbf{x}_{i, j}^{t}+\mathbf{x}_{i-1, j}^{t}$ and choosing $\alpha^{t}(i, j)=4 \beta_{i j}^{t}+1-\gamma_{i j}^{t}$, one can obtain the following iterative formula.

$$
\begin{aligned}
\mathbf{x}_{i, j}^{t+1}= & \frac{1}{4 \beta_{i j}^{t}+1-\gamma_{i j}^{t}}\left[\beta_{i j}^{t}\left(\mathbf{x}_{i+1, j}^{t}+\mathbf{x}_{i-1, j}^{t}+\mathbf{x}_{i, j+1}^{t}+\mathbf{x}_{i, j-1}^{t}\right)\right. \\
& \left.+\left(1-\gamma_{i j}^{t}\right) \mathbf{x}_{i j}^{\prime t}+\gamma_{i j}^{t} N_{i j}^{t}\right]
\end{aligned}
$$

When the surface is far away from the cortex, $\gamma_{i j}^{t}$ is equal to one, so that the iterative scheme is similar to the Point Jacobi Method [12]. Although it is possible to use a faster convergent scheme, such as the Successive Overrelaxation scheme used by [8], we found that for a large number of nodes, some of them might move among others, so that the distance homogenisation step, described in the next section, cannot be performed without making errors, due to local folding-over of the surface. Performing the distance homogenisation after each iteration, provides the additional advantage, that since the distances between the nodes remain more or less the same, the second order derivative, will be better approximated in the next step, than when the nodes are spread non-uniformly over the surface.

\subsection{Homogenising Distances}

After each deformation step, the distances between the points are homogenised by an enhanced version of the Fixed-Point algorithm described in [8], to achieve a faithful visualisation of cortical anatomy later on. As stated before in the introduction, the optimal mapping is the isometric mapping, which preserves distances and angles everywhere. From differential geometry, we know that a mapping $\left\{u^{\prime}=u^{\prime}(u, v) ; v^{\prime}=v^{\prime}(u, v)\right\}$ is isometric when the first fundamental forms are identical at two corresponding points: $d s^{2}=E(u, v) d u^{2}+2 F(u, v) d u d v+$ $G(u, v) d v^{2}=E^{\prime}\left(u^{\prime}, v^{\prime}\right) d u^{\prime 2}+2 F^{\prime}\left(u^{\prime}, v^{\prime}\right) d u^{\prime} d v^{\prime}+G^{\prime}\left(u^{\prime}, v^{\prime}\right) d v^{\prime 2}=d s^{\prime 2}$

Two surfaces are locally isometric, if there is a local isometry at every point for each of the surfaces. However a local isometry may fail to be an isometry, 
when the surfaces have different topological properties (e.g. the cylinder and the plane, or the cortical surface and the plane). Even establishing a local isometry everywhere between the cortical surface and the plane is an impossible task, which is also true for the homothetic mapping, introduced in [8] to relax the condition on the distances, by requiring that they are preserved up to a global constant $C$ : i.e. $E=C \cdot E^{\prime}, F=C \cdot F^{\prime}, G=G \cdot E^{\prime}$. Note however, that once the homothetic mapping is known, the isometric mapping with the plane can be easily obtained by uniformly scaling the $(u, v)$-plane with $C^{-1}$. Since the fundamental form coefficients in the plane are $E=1, F=0$ and $G=1$, the ideal homothetic mapping with the cortical surface is given by $E^{\prime}=C, F^{\prime}=0$ and $G^{\prime}=C$, in any point. As $E=\mathbf{x}_{u} \cdot \mathbf{x}_{u}, F=\mathbf{x}_{u} \cdot \mathbf{x}_{v}$ and $G=\mathbf{x}_{v} \cdot \mathbf{x}_{v}, \mathbf{x}\left(u, v_{0}\right)$ and $\mathbf{x}\left(u_{0}, v\right)$ will be constant speed curves, orthogonal to each other.

In [8], a regularisation of the positions of the nodes of the surface is obtained via alternatively adjusting all curves for constant $v=v_{0}$, i.e. $\mathbf{x}\left(u, v_{0}\right)$, and all curves for constant $u=u_{0}$, i.e. $\mathbf{x}\left(u_{0}, v\right)$ to make them equidistant between the nodes. An additional procedure is used to make the curves orthogonal to each other. We found that this heuristic approach, performs even better when the additional orthogonalisation step is replaced by two extra regularisation or homogenisation steps along both diagonal directions.

In our approach, the actual displacement $\Delta \mathbf{x}(u, v)$ of the nodes is only performed, when all the four regularisation steps have been accomplished and not after each step separately. It is the average of the four separate displacements: i.e. $\Delta \mathbf{x}(u, v)=\frac{1}{4} \sum_{k=1}^{4} \Delta \mathbf{x}_{k}(u, v)$. In order to ensure that the points move along the surface, the displacement is restricted to the tangent plane:

$$
\Delta \mathbf{x}_{t p}(u, v)=\Delta \mathbf{x}(u, v)-\frac{<\Delta \mathbf{x}(u, v), \mathbf{N}(u, v)>}{|\boldsymbol{N}(u, v)|^{2}} \cdot \mathbf{N}(u, v)
$$

The above steps are repeated a number of times and we stop when all $\Delta \mathbf{x}_{t p}(u, v)$ are less than a certain tolerance factor, or the maximum number of iterations has been reached.

\subsection{The Coarse to Fine Approach}

Once the surface has reached its final position, curves and nodes are added via interpolation to form a fine mesh. This fine mesh is deformed via the same strategy, as described in the sections 2.3 and 2.4, until it reaches its equilibrium state.

\subsection{Shading the Cortical Surface}

When the elastic deformation process has converged, a ray is fired for each point normal to the obtained dilated surface (defined in section 2.1). At the intersection of these rays with the original segmented brain, we compute the shading analogous to volume rendering and attribute the result to the corresponding $(u, v)$ point in the plane. The planar images of both the upper and lower surface 
are laid next to one another and provide as such a continuous flat projection of the complete cortex. The formula we apply is a weighted sum of a contrast enhanced depth-shading term and a Phong-shading term[13].

$$
\begin{aligned}
& I=\alpha(d c)^{\gamma}+(1-\alpha) \cdot\left(I_{a} k_{a}+d c \cdot I_{l}\left(k_{d}\left(\boldsymbol{N}_{\text {org }} \cdot \boldsymbol{L}\right)+k_{s}(\boldsymbol{R} \cdot \boldsymbol{V})^{n}\right)\right) \\
& \text { with } d c=\left(1-K \frac{d e p t h}{\text { depth }_{M A X}}\right)
\end{aligned}
$$

Since we compute a grey level intensity image only, we consider the ambient intensity $I_{a}$, the intensity $I_{l}$ of the light source and the material constants $k_{a}$ (ambient), $k_{d}$ (diffuse), $k_{s}$ (specular) to be the same for the three colour primaries $(\mathrm{R}, \mathrm{G}, \mathrm{B})$. Further in this formula $\boldsymbol{N}_{\text {org }}$ stands for the normal at the intersection point with the original cortical surface, $\boldsymbol{L}$ is the direction of the light source, $\boldsymbol{R}$ is the reflection vector and $\boldsymbol{V}$ is the vector pointing towards the observer. The exponent $n$ determines the spread of the specular reflections $(n \rightarrow \infty$ corresponds to the perfect mirror). Since, we have to compute the shading from every direction, it is better not to fix the position / direction of the observer and the light source in a global way, but to locally vary them for each element $(u, v)$ that is shaded. A possible way of implementing this, is to assume that both $\boldsymbol{V}$ and $\boldsymbol{L}$, are oriented along the direction of the inner normal of the dilated surface $\boldsymbol{N}_{\text {dil }}(u, v)$. The inner product $\boldsymbol{R} \cdot \boldsymbol{V}$ then simplifies to $\boldsymbol{R} \cdot \boldsymbol{L}=2\left(\boldsymbol{N}_{\text {org }} \cdot \boldsymbol{L}\right)^{2}-1$. $\boldsymbol{N}_{\text {org }}(u, v)$ is computed via the grey-level gradient of the binary image [13].

In order to obtain useful results with the depth shading approach, we apply a non-linear contrast enhancement method based on $\gamma$-correction, since depth variations are usually very small, due to the fact that they are measured from the nearby dilated surface.

\section{Results}

Fig. 2 demonstrates the feasibility of the deformable surface and distance homogenisation approach. The results are produced for a $256 \times 256 \times 115 \mathrm{~T} 1$ weighted MR set, that has been interpolated to obtain a $282 \times 388 \times 230$ volume. This operation is not really mandatory (other acceptable projections were obtained for a number of volume sets without interpolation). A grid of $361 \times 401$ points is defined in both $(u, v)$ planes.

By connecting the $(u, v)$ nodes in addition along one diagonal direction, one can directly obtain a triangular mesh for the mapped $\mathbf{x}(u, v)$ points at the surface. Fig. 2(a) shows a surface shaded view from the top, generated by means of a triangular mesh deformed towards the original cortical surface. A regular sample of constant speed curves has been overlaid on the surface. Note that distances and orthogonality are preserved rather well. The regularity of mapped nodes can also be directly inspected from the triangular mesh itself. Fig. 2(b) shows a very fine mesh of the dilated cortical surface. Even the very fine mesh is free from folded-over triangles, a problem that arises when the homogenisation pass is not performed after each deformation step. 
Fig. 3 and Fig. 4 show renderings obtained for the entire unfolded cortex. Note that sulci can indeed be followed in their entirety. During our experiments we found that the Phong Shading component leads to very thick sulci, while pure depth shading might not visualise the smaller sulci well. By interactively adjusting the parameter $\alpha$, the user can blend both results until he obtains a visually appealing and well interpretable image.

Currently with this number of points $722 \times 401$ and volume size $282 \times 388 \times 230$, such a rendering can be produced in about 5 minutes on a Pentium II $300 \mathrm{MHz}$, the interactive definition of the hemi-ellipsoids included. The deformation, including node regularisation, towards the dilated surface of a coarse grid consisting of $74 \times 41$ points, mapped initially on the hemi-ellipsoids, is performed in about 15 sec. Five extra steps of deformation and regularisation for the complete grid of $722 \times 401$ nodes takes about 3 minutes. After these steps, a well fitted surface is obtained for the dilated cortex. From this surface the rendering can be made in $4 \mathrm{sec}$.

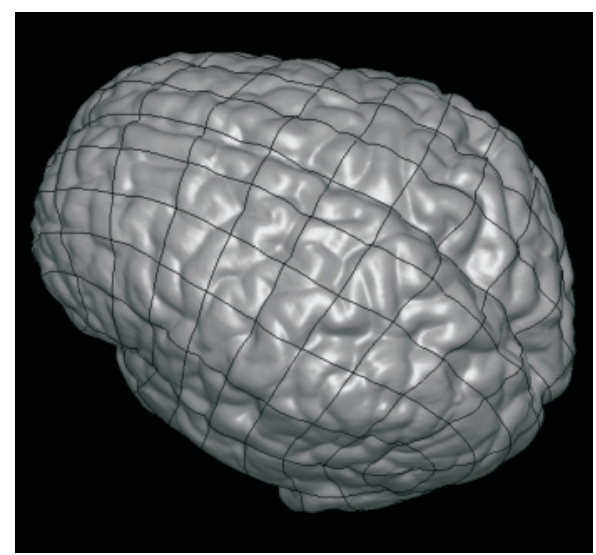

(a)

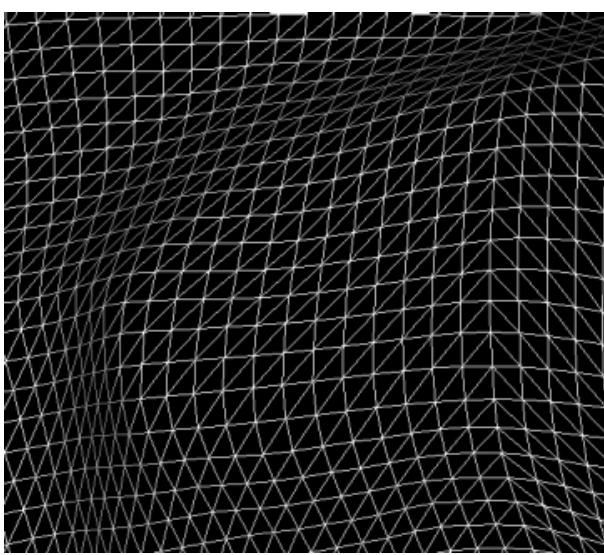

(b)

Fig. 2. Overlay of a sample of the constant speed curves on the original cortical surface (a) and The detailed mesh of a restricted area of the dilated cortical surface (b)

\section{Discussion}

The method proves to be quite robust. Its clinical value is currently evaluated. Since it takes only about 5 minutes to produce the rendering, it could even be envisaged to use such a kind of visualisation in clinical routine. Until now, we did not optimise the deformation and homogenisation algorithms in terms of computer instructions and register usage. Further, it is possible to consider a 


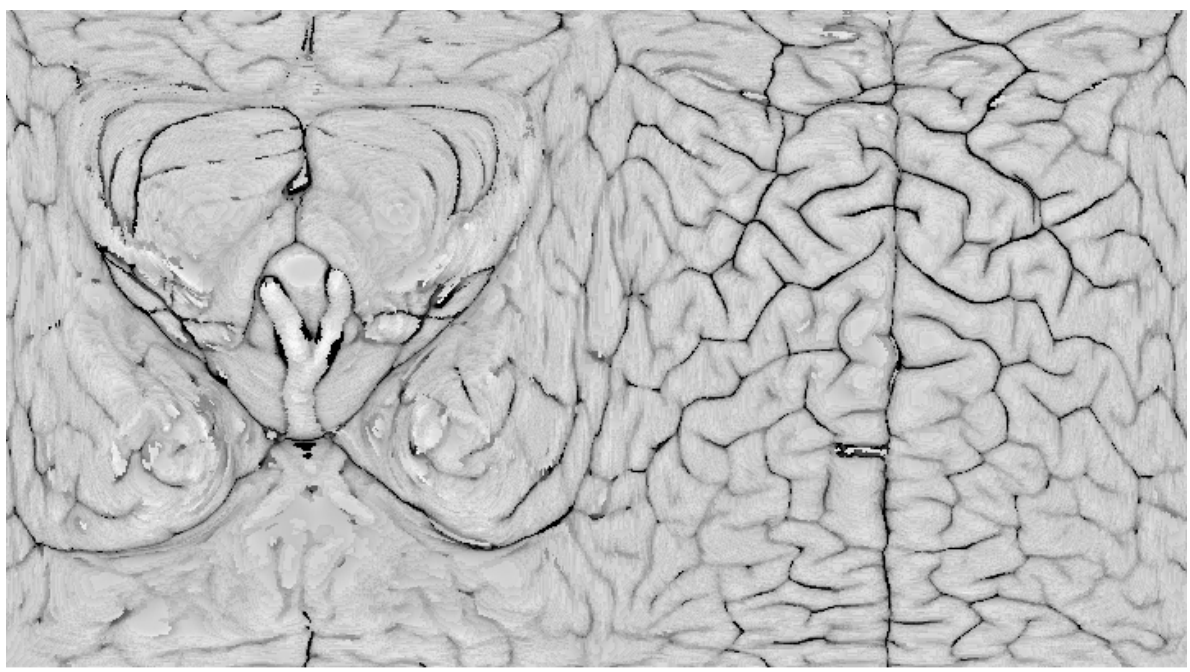

Fig. 3. Rendering of the unfolded cortical surface (emphasis on depth shading) $\alpha=1.0, \gamma=1, K_{a}=0.1, K_{d}=0.4, K_{s}=0.5, K=1$, depth MAX $_{A}=15$.

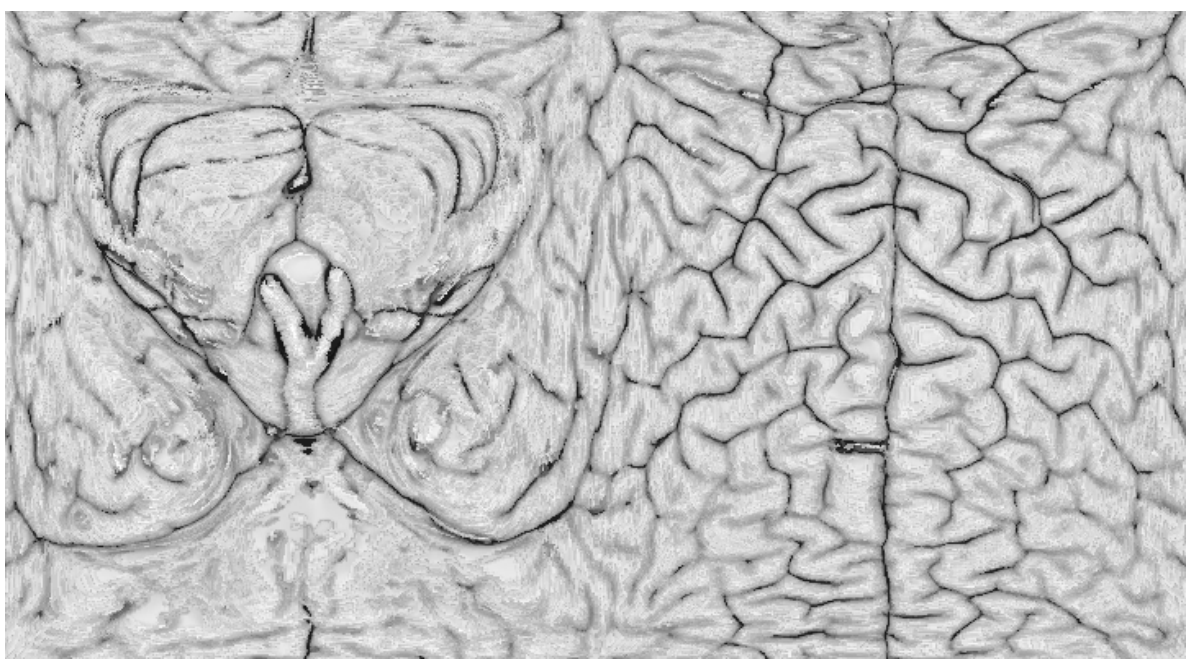

Fig. 4. Rendering of the unfolded cortical surface (depth and Phong shading) $\alpha=0.7, \gamma=1, K_{a}=0.1, K_{d}=0.4, K_{s}=0.5, K=1$, depth MAX $_{A}=15$. 
supplementary mesh, in between the coarse and fine mesh to further reduce the computation time. In addition one could try to derive the position and the size of the hemi-ellipsoids in an automatic way. While the approach could lead to a new practice to examine, analyse and visualise cortical anatomy, it also has the additional advantage to produce an extremely regular surface mesh of the cortical surface at any resolution (1.000 to 1.000 .000 triangles) required by the user. Such a mesh could be used in finite-element studies or just for surface visualisation purposes (e.g. visualising the loci at the surface during stimulation studies).

\section{Acknowledgements}

This research was funded by a bilateral scientific and technological co-operation "Flanders-China" / Grant BIL97/72. The authors are very grateful to Prof. Pengfei Shi and Prof. Xin Yang for their co-operation in the organisation of scientific exchanges between Shanghai Jiaotong University and VUB.

\section{References}

1. D. Hubel and T. Wiesel. Laminar and columnar distribution of geniculo-cortical fibers in the macaque monkey. J Comp Neurol, 146:421-450, 1972. 287

2. D. Van Essen and S. Zeki. The topographic organization of rhesus monkey prestriate cortex. J Physiol (Lond), 277:193-266, 1978. 287

3. A. Dale and M. Sereno. Improved localization of cortical activity by combining EEG and MEG with MRI cortical surface reconstruction: a linear approach. $J$ Cognit Neurosci, 5:162-176, 1993. 287

4. E. Schwartz, A. Shaw, and E. Wolfson. A numerical solution to the generalized mapmaker's problem: flattening nonconvex polyhedral surfaces. IEEE Trans Pattern Anal Machine Intell, 11:1005-1008, 1989. 288

5. E. Wolfson and E. Schwartz. Computing minimal distances on arbitrary twodimensional polyhedral surfaces. IEEE Pattern Anal Machine Intell, 11:1001-1005, 1989. 288

6. D. Tombeur. Investigations into Unitary and Non-Euclidean Geometry of Biomedical Images. Object Oriented Technology for Image Manipulation. PhD Thesis, Vrije Universiteit Brussel, 1994. 288

7. G. Carman, H. Drury, and D. Van Essen. Computational Methods for Reconstructing and Unfolding the Cerebral Cortex. Cerebral Cortex, 5:506-517, 1995. 288

8. C. Davatzikos and R. Bryan. Using a deformable surface model to obtain a shape representation of the cortex. IEEE Trans. on Medical Imaging, 15(6):785-795, 1996. 288, 289, 290, 291, 292

9. D. Van Essen and H. Drury. Structural and Functional Analyses of Human Cerebral Cortex Using a Surface-Based Atlas. The Journal of Neuroscience, 17(18):70797102, 1997.288

10. A. Salomie, E. Nyssen, and J. Cornelis. Multivariate Techniques for Medical Image Segmentation. Proceedings of the Signal Processing Symposium SPS 98, Leuven, March 26-27, 155-158, 1998. 289 
11. L. Cohen and I. Cohen. Finite element methods for active contour models and balloons for 2D and 3D images. IEEE Trans Pattern Anal Machine Intell, 15(11):1131-1147, 1993. 289

12. C. Hirsch. Numerical Computation of Internal and External Flows. Volume 1: Fundamentals of Numerical Discretization, Wiley, New-York, 1989. 291

13. U. Tiede, K. Höhne, M. Bomans, A. Pommert, M. Riemer, and G. Wiebecke. Investigation of Medical 3D-Rendering Algorithms. IEEE Computer Graphics 86 Applications, 41-52, 1990. 293 